\title{
CUIDADOS À PESSOA SUSPEITA DE COVID-19 COM SINAIS DE GRAVIDADE NAATENÇÃO PRIMÁRIA Á SAÚDE
}

Patricia Madalena Vieira Hermida Natálie D'Avila da Silveira ${ }^{1}$ Lucas Jose Bringhenti ${ }^{1}$ Tatiane Shimanko Bugs ${ }^{1}$ Paola Miottol Meriane Ferrarezi Chiari ${ }^{1}$ Paola Miranda Sulis ${ }^{1,2}$

\begin{abstract}
https:// orcid.org/0000-0002-7969-357X https:// orcid.org/0000-0003-0340-5196 https:// orcid.org/0000-0002-2228-8733 https:// orcid.org/0000-0001-7355-3368 https:// orcid.org/0000-0002-0134-9728 https:// orcid.org/0000-0002-7755-7267 https:// orcid.org/0000-0002-2027-2471
\end{abstract}

Objetivo: Descrever o processo de elaboração e a implementação de um checklist de cuidados à pessoa suspeita do novo coronavírus com sinais de gravidade na Atenção Primária à Saúde. Métodos: Relato de experiência de um Centro de Saúde da região da Grande Florianópolis, Santa Catarina, desenvolvida em duas etapas: elaboração e implementação de um checklist de cuidados para pessoas com sintomas respiratórios graves. Resultados: O checklist, elaborado coletivamente por enfermeiros e médicos em quatro versões, constituiu-se, na sua versão final dos itens: sinais vitais, ventilação, procedimentos, medicação, biossegurança, cuidados com a família, alergia medicamentosa, comunicação com outros pontos da Rede de Atenção à Urgência e observações. Implementado no atendimento a uma pessoa com sintomas respiratórios graves suspeita do novo coronavírus, o checklist proporcionou maior segurança no cuidado, acesso rápido às informações e com garantia de que nenhum dado fosse negligenciado, ademais favoreceu o diálogo entre os profissionais durante o atendimento, a comunicação com a família e com outros pontos da Rede de Atenção às Urgências. Conclusões: O checklist elaborado, claro e objetivo na sua implementação, supriu a necessidade de se garantir, na Atenção Primária à Saúde, uma assistência à pessoa suspeita do novo coronavírus com sinais de gravidade com mais qualidade e segurança.

Descritores: Enfermagem; Lista de Checagem; Infecções por Coronavírus; Segurança do Paciente; Atenção Primária à Saúde.

\section{CARE OF SUSPECTED PERSON OF COVID-19 WITH SEVERE SIGNS IN PRIMARY HEALTH CARE}

Objective: To describe the elaboration process and implementation of a care checklist to suspected persons of the new coronavirus with severe signs in Primary Health Care. Methods: Report of experience of a health center in the region of the greater Florianópolis, Santa Catarina, developed in two phases: elaboration and implementation of a checklist for care of people with severe respiratory symptoms. Results: The checklist was elaborated collectively by nurses and physicians in four versions, and its final version consists of the items: vital signs, ventilation, procedures, medication, biosafety, family care, drug allergy, communication with other branches within the Urgent Care Network, and observations. Implemented in the care of persons with severe respiratory symptoms suspected of the new coronavirus, the checklist provided greater safety in care, quick access to information and it guaranteed that no data was neglected, in addition it facilitated the dialogue between professionals during the service, the communication with the family and with other branches within the Urgent Care Network. Conclusions: The elaborated checklist was clear and objective in its implementation, and fulfilled the need to guarantee more quality and safety in assistance of suspected person of the new coronavirus with severe signs in Primary Health Care.

Descriptors: Nursing; Checklist; Coronavirus Infections; Patient Safety; Primary Health Care.

\section{CUIDADOS A LA PERSONA SOSPECHOSA DE COVID-19 CON SIGNOS DE GRAVEDAD EN LAATENCIÓN PRIMARIA DE SALUD}

Objetivo: Describir el proceso de elaboración y la implementación de un checklist de cuidados a la persona sospechosa del nuevo coronavirus con signos de gravedad en la Atención Primaria de Salud. Método: Relato de experiencia de un Centro de Salud de la región de la Grande Florianópolis, Santa Catarina, desarrollada en dos etapas: elaboración e implementación de un checklist de cuidados para personas con síntomas respiratorios severos. Resultados: El checklist elaborado colectivamente por enfermeras y médicos en cuatro versiones, consistió en su versión final de los ítems: signos vitales, ventilación, procedimientos, medicamentos, bioseguridad, cuidados con la familia, alergia a medicamentos, comunicación con otros puntos de la Red de Atención de Urgencia y observaciones. Implementado en la atención de una persona con síntomas respiratorios severos y sospechosa del nuevo coronavirus, el checklist proporcionó una mayor seguridad en el cuidado, acceso rápido a la información y garantía que ningún dato fuera desestimado. Además favorecer el diálogo entre profesionales durante la atención, la comunicación con la familia y con otros puntos de la Red de Atención de Urgencias. Conclusiones: El checklist elaborado, claro y objetivo en su implementación, cumplió la necesidad de garantizar en la Atención Primaria de Salud, la asistencia a la persona sospechosa del nuevo coronavirus con signos de gravedad con más calidad y seguridad. Descriptores: Enfermería; Lista de Verificación; Infecciones por Coronavirus; Seguridad del Paciente; Atención Primaria de Salud.

1Secretaria Municipal de Saúde de Florianópolis, SC, Brasil.

${ }^{2}$ Centro Universitário Estácio, SC, Brasil.

Autor Correspondente: Patrícia Madalena Vieira Hermida E-mail - patymadale@yahoo.com.br

Recebido: $26 / 7 / 20$ Aceito: 06/8/20 


\section{INTRODUÇÃO}

O mundo acompanha a evolução da infecção pelo novo coronavirus, denominado SARS-CoV-2, causador da doença conhecida como COVID-19. Os primeiros casos surgiram no final de dezembro de 2019, na cidade de Wuhan na China, sugerindo uma transmissão inicial de animais para humanos, mas que logo tomou proporções intercontinentais com a evidência de transmissão sustentada entre humanos. Essa transmissão ocorre, sobretudo, por meio do contato de pessoas saudáveis com gotículas respiratórias ou aerossóis provenientes de uma pessoa contaminada, por meio da respiração ou contato com mucosas, como nariz e boca. Diferentemente dos já conhecidos vírus da família dos coronavírus, o SARS-CoV-2 se caracteriza por alta taxa de transmissibilidade e sintomas que podem variar de quadros leves, em sua grande maioria, até formas graves, caracterizadas pela Síndrome Respiratória Aguda Grave (SRAG) ${ }^{1}$.

Em decorrência dessa infecção causada pelo novo coronavírus (2019-nCoV) no Brasil, o Ministério da Saúde publicou a Portaria no 188/2020, declarando Emergência de Saúde Pública de Importância Nacional (ESPIN) e instituiu o Centro de Operações de Emergências em Saúde Pública (COE-nCoV) como mecanismo de gestão coordenada da resposta à emergência no âmbito nacional ${ }^{2}$

No país, a Atenção Primária à Saúde (APS), representada sobretudo pela Estratégia de Saúde da Familia (ESF), tem papel essencial no combate à COVID-19, como já evidenciado em outros surtos e pandemias. Considerada porta de entrada do Sistema Único de Saúde (SUS), a APS é responsável pela identificação precoce das pessoas suspeitas da doença, com manejo de casos graves e encaminhamento destes para os serviços especializados $^{3}$, o que requer dos profissionais da saúde o reconhecimento da SRAG.

A SRAG, principal complicação decorrente da infecção pelo coronavírus, trata-se de uma condição que pode acometer pessoas de qualquer idade que apresentem febre alta (mesmo que referida), tosse e dispneia, acompanhadas de outros sinais de gravidade como: saturação de oxigênio (SpO2) <95\% em ar ambiente; sinais de desconforto respiratório ou taquipneia; hipotensão e piora nas condições clínicas das doenças de base. Nas crianças também pode ocorrer batimentos de asas nasal, cianose, tiragem intercostal, desidratação e inapetência. Cabe ressaltar que nem todas as pessoas apresentam febre, a exemplo de crianças, idosos, imunossuprimidos e aquelas em uso de antitérmicos ${ }^{3}$.

Nesse cenário se encontra inserida a Enfermagem, que desempenha papel relevante na detecção e avaliação dos casos suspeitos do novo coronavírus, pela sua capacidade técnica e por constituir o maior quantitativo de profissionais da área da saúde, além de ser a única categoria profissional que permanece
24 horas junto à pessoa doente 4 . O surgimento de novas doenças e as constantes transformações na área da saúde desafiam os envolvidos no cuidado, sobretudo à equipe de Enfermagem, que tem no SUS o seu principal lócus de atuação e que requer boas práticas profissionais para uma assistência segura e de qualidade. Entende-se como boas práticas em Enfermagem o conjunto (inter-relacionado e indissociável) de teorias, técnicas, processos e atividades vistas como as melhores opções disponiveis para o cuidado, que possui consistência com conhecimentos, valores, contextos, ambientes, objetivos e evidências no interesse da saúde ${ }^{5}$.

Nesse meandro, destaca-se o uso de listas de verificação (checklists), uma vez que otimizam o cuidado na atenção à saúde, organizam a rotina e as tarefas da equipe e garantem que nenhuma demanda seja esquecida. Ademais, contribuem para melhorar a comunicação entre equipes e garantir que informações importantes sejam lembradas ${ }^{6}$.

Diante do exposto, considerando a relevância de boas práticas de Enfermagem no enfrentamento ao novo coronavírus nos serviços públicos de saúde, o presente estudo teve como objetivo descrever o processo de elaboração e implementação de um checklist de cuidados à pessoa suspeita do novo coronavírus com sinais de gravidade na Atenção Primária à Saúde.

\section{MÉTODO}

\section{Tipo de Estudo}

Trata-se de um relato de experiência sobre a elaboração e implementação de um checklist de cuidados à pessoa suspeita de COVID-19 que apresenta sinais de gravidade na APS.

\section{Cenário do Estudo}

A experiência ocorreu em um Centro de Saúde (CS) de um bairro periférico de um município da Grande Florianópolis - SC. O CS possui cinco equipes da ESF, cada uma composta por Médico de Família, Enfermeiro, Técnico de Enfermagem e Agente Comunitário de Saúde. A unidade recebe o Programa de Residência de Medicina de Família e Comunidade e o Multiprofissional em Saúde da Família, contando com três residentes médicos, uma de Enfermagem, de Nutrição e de Farmácia. Conta também com uma equipe de Saúde Bucal, constituída por cirurgião-dentista e técnico de saúde bucal, e uma equipe do Núcleo Ampliado de Saúde da Família (NASF) com profissionais de Fisioterapia, Nutrição, Serviço Social e Educação Física.

Período de realização da experiência

A experiência ocorreu durante os meses de abril à junho de 2020, quando foram desenvolvidas a elaboração e implementação do checklist.

\section{Sujeitos envolvidos na experiência}

Participaram efetivamente da elaboração do instrumento cinco enfermeiros, dois médicos, uma residente de Enfermagem 
e dois residentes de Medicina. Adotou-se como critérios de inclusão dos participantes: ser enfermeiro, médico de família ou residentes destas categorias, atuantes no CS, independente do tempo de atuação no serviço e da idade dos profissionais. Justifica-se que a escolha dos participantes levou em consideração o fato das pessoas suspeitas de COVID-19 serem atendidas no CS exclusivamente por enfermeiros, médicos ou residentes de tais categorias.

\section{Aspectos Éticos}

Neste estudo foi garantido o anonimato aos profissionais que colaboraram para a elaboração e implementação do checklist e, por se tratar da experiência dos autores, este dispensa aprovação do Comitê de Ética em Pesquisa.

\section{Objetivos da Experiência}

Considerando-se o cenário de pandemia instalado e a APS como porta preferencial de entrada do SUS e ponto da Rede de Atenção às Urgências (RAU), esta experiência teve como objetivo: Desenvolver um instrumento prático, objetivo, técnico e direcionador de cuidados às pessoas suspeitas de COVID-19 com sinais de gravidade atendidas em um Centro de Saúde; otimizar com a implementação de um instrumento a assistência prestada às pessoas suspeitas de COVID-19 com sinais de gravidade em um território da Estratégia de Saúde da Família.

\section{Descrição da Experiência}

Para o atendimento de pessoas com sintomas respiratórios é destinada uma sala específica do CS, com entrada e local de espera própria, separada daquela voltada para os demais atendimentos da unidade de saúde, como recomendação da Diretoria de Atenção Primária em Saúde do Município. Essas pessoas são atendidas por um médico e um enfermeiro do CS, que se revezam com outros profissionais das mesmas categorias, conforme escala de trabalho. Nessa sala estão disponiveis todos os materiais e Equipamentos de Proteção Individual (EPI) necessários para o suporte básico às urgências decorrentes de SRAG, atendimento prestado até que o Serviço de Atendimento Móvel à Urgência e Emergência Móvel (SAMU) realize a transferência da pessoa para o hospital de referência aos casos suspeitos ou confirmados de COVID-19.

A experiência deste estudo ocorreu em duas etapas. A primeira refere-se à elaboração do checklist de cuidados, que teve início em abril de 2020, após o CS realizar o primeiro atendimento a uma pessoa com sinais de gravidade suspeita do novo coronavírus. Essa etapa foi concluída ainda em abril, após a produção coletiva da última versão do instrumento. A segunda etapa de experiência trata-se da implementação do checklist por uma das enfermeiras, em junho de 2020, quando o instrumento foi aplicado no atendimento do segundo caso de pessoa suspeita de COVID-19, com sinais de gravidade.

Elaboração do Checklist
A primeira versão do instrumento, elaborada pela residente de Enfermagem, foi produzida a partir da experiência dos profissionais no atendimento do primeiro caso de pessoa com urgência respiratória suspeita do novo coronavírus, com base nas "Orientações para manejo do paciente com suspeita de COVID-19 e com sintomas respiratórios graves na APS"7 , desenvolvidas pela Secretaria da Saúde do município em questão.

Essa versão do checklist se constituiu dos itens: identificação (nome, data de nascimento, data do atendimento, data dos primeiros sintomas, caso suspeito/em investigação/confirmado); sinais vitais (frequência cardiaca, saturação de oxigênio, frequência respiratória, temperatura corporal e pressão arterial); ventilação (ar ambiente, óculos nasal, máscara ou outro e ausculta pulmonar); acesso venoso (membro superior direito, membro superior esquerdo ou outro, salinizado, soro fisiológico, medicamentos); medicação (nome e hora de administração); biossegurança (limpeza de materiais, troca de máscara, uso de EPI e discriminação de quais); contato com familiares (não, sim e com quem falou); observações (sinais e sintomas, encaminhamentos).

Elaborada, a primeira versão do checklist foi postada em um grupo vinculado a um aplicativo de mensagens, utilizado formalmente no CS como forma de comunicação entre os médicos, enfermeiros e residentes destas categorias. No grupo ocorreram discussões e sugestões dos profissionais para melhorias no instrumento, as quais geraram outras versões do checklist. As adequações em cada versão foram realizadas pela residente de enfermagem.

Na segunda versão, conforme sugestão dos participantes retirou-se os dados de identificação por se tratar de um checklist e houve a mudança da denominação do item "Acesso Venoso" por "Procedimentos", incluindo-se neste a opção de sondagem vesical de demora (sim ou não), pois entende-se que, a depender da gravidade da pessoa, esta já poderia ser transferida com a sonda instalada para favorecer o equilibrio hemodinâmico.

$\mathrm{Na}$ terceira versão as alterações realizadas a partir das recomendações dos participantes foram: acréscimo na informação do horário da aferição dos sinais vitais e Hemoglicoteste (HGT), para melhor controle da evolução e condições clínicas da pessoa. O item "Contato com Familiares" tornou-se "Cuidado com a Família", no qual se acresceu orientações sobre o isolamento domiciliar, a fim de lembrar das recomendações fundamentais aos familiares contatos de caso confirmado ou suspeito. Além disso, incluiu-se outros dois itens: "alergia medicamentosa", na busca pela segurança do paciente; e "Contatos Externos" (SAMU e hospital de referência), para orientar o profissional que está realizando o atendimento sobre o fluxo de continuidade do cuidado na RAU. Os demais itens (ventilação, procedimentos, medicação, biosse- 
gurança e observações) não sofreram alterações.

Em sua quarta e última versão, realizou-se outras adequações no checklist, sobretudo relacionadas à sequência e nomenclatura de itens, para melhor organização e caracterização destes. Assim, o item "Alergia medicamentosa" foi posicionado logo abaixo dos itens "Biossegurança" e "Cuidados com a família". O item "Contatos Externos" passou a ser denominado "Comunicação com outros pontos da Rede de Atenção às Urgências" e adicionado neste o "Encaminhamento impresso ao hospital", excluindo-se "Encaminhamentos" que constava em "Observações". Ademais, ainda foram acrescentados em "Procedimentos", no acesso venoso, o tipo de dispositivo (abocath ou scalp e respectivo número), o que pode ser importante na continuidade do cuidado em outros pontos da rede de atenção. Em "Cuidados com a Família", inseriu-se a orientação de restrição domiciliar junto à orientação do isolamento domiciliar, uma vez que para contatos assintomáticos a recomendação é de restrição ao invés de isolamento. A versão final do checklist proposto no presente estudo está apresentada na Figura 1.

Figura 1 - Checklist de cuidados à pessoa suspeita de COVID com sinais de gravidade. Grande Florianópolis, SC, 2020.

CUIDADOS A PESSOA SUSPEITA DE COVID COM SINAIS DE GRAVIDADE

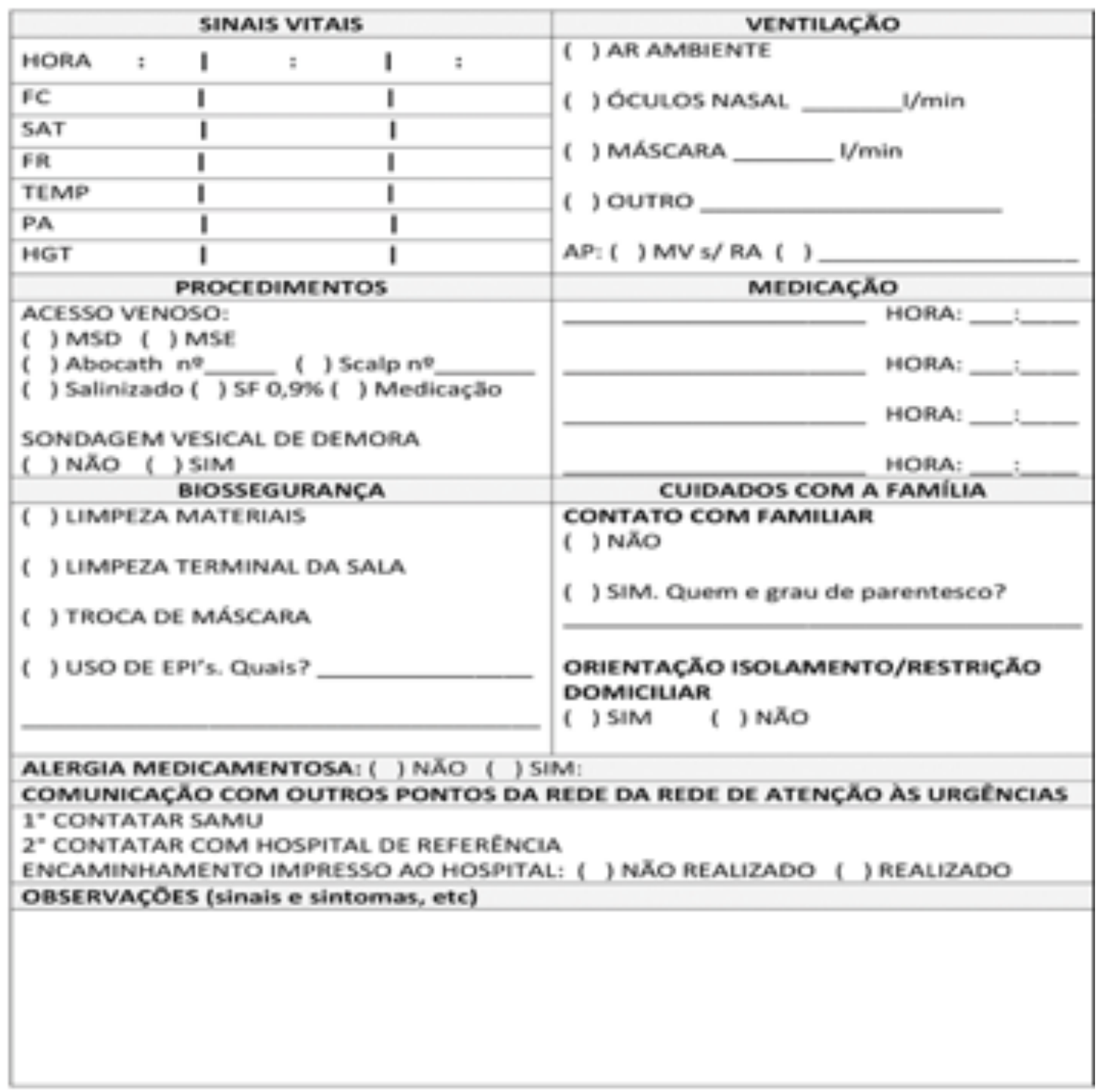

Nota: FC: Frequência Cardiaca; SAT: Saturação de Oxigênio; FR: Frequência Respiratória; TEMP: Temperatura corporal; PA: Pressão Arterial; HGT: Hemoglicoteste; AP: Ausculta Pulmonar; MV s/ RA: Murmúrios Vesiculares sem Ruídos Adventícios; MSD: Membro Superior Direito; MSE: Membro Superior Esquerdo; SF 0,9\%: Soro Fisiológico 0,9\%; EPI: Equipamento de Proteção Individual. 
A elaboração do checklist proporcionou uma importante discussão entre a equipe, fomentando a revisão e atualização de conhecimentos. Entretanto, nem todos participaram em momento oportuno das discussões, o que pode estar relacionado à periodicidade com que os profissionais acessam o aplicativo de mensagens utilizado no processo de construção do instrumento.

\section{IMPLEMENTAÇÃO DO CHECKLIST}

Aplicou-se o checklist no atendimento a uma pessoa sintomática respiratória grave, suspeita de COVID-19, momento em que todos os itens do instrumento foram checados e que resultou no registro de dados. Apenas o item "medicação" não produziu dados, uma vez não houve administração de medicamentos. $O$ fato de o atendimento ter sido realizado pelo mesmo enfermeiro que atendeu previamente outra pessoa sintomática respiratória grave sem o uso do instrumento, pois este ainda não existia, favoreceu a análise da sua implementação e a identificação dos resultados alcançados.

O enfermeiro que implementou o checklist não teve dificuldades em utilizá-lo, talvez pelo fato deste ter participado a sua elaboração. Todavia julga-se necessário uma aproximação prévia com o instrumento, a partir da educação permanente da equipe, para que seja aplicado com rapidez e segurança em cenários semelhantes.

A elaboração e implementação do checklist no atendimento de pessoas suspeitas de COVID-19 com sinais de gravidade na APS, no contexto de um CS do município em questão, levou em consideração o provável aumento do número de casos de SRAG, devido à vigência da pandemia causada pelo novo coronavírus no ano 2020.

De modo geral, estima-se que dentre os casos confirmados da doença, 20\% requerem cuidados mais complexos e, mais especificamente, $5 \%$ exijam cuidados intensivos de saúde. Esses dados implicam no aumento do número de atendimentos a pacientes com sintomas respiratórios em toda rede de saúde, inclusive na APS, porta de entrada do SUS ${ }^{8}$. Nesse meandro, a Política Nacional de Atenção às Urgências considera as Unidades Básicas de Saúde, as quais representam a APS no país, unidades de atendimento pré-hospitalar de modalidade fixa, enquanto o SAMU configura o atendimento pré-hospitalar móvel, responsável pela atenção intermediária de saúde juntamente com as Unidades de Pronto Atendimento (UPA). A atenção às urgências que requerem serviços de maior densidade tecnológica, são encaminhadas para unidade hospitalares de média e alta complexidade?

Apesar do exposto, as unidades de saúde não neces- sariamente estão preparadas para a atenção às urgências, assim como os profissionais. Estudo revelou dificuldades de equipes da APS no atendimento às urgências relacionadas especialmente à estrutura física, recursos materiais e qualificação profissional insuficientes ${ }^{10}$. Do mesmo modo, outro estudo evidenciou que apesar da enfermagem ter papel fundamental nos cuidados de saúde em urgência e emergência, o que exige dos profissionais reconhecimento quase imediato da condição do paciente e manejo adequado, nem sempre estes se sentem seguros e preparados para o enfrentamento dessas situações, especialmente devido à inexperiência associada à falta de estrutura e prática profissional ${ }^{11}$

No contexto deste relato de experiência, a necessidade de reorganização do serviço de saúde para atender às pessoas com suspeita de infecção pelo novo coronavírus se deu a partir do primeiro caso de SRAG atendido pelos profissionais da APS. A construção coletiva e dinâmica do checklist mobilizou os profissionais envolvidos no cuidado e propiciou avaliar as práticas profissionais.

O checklist apresentado neste estudo contém itens que evidenciam a preocupação dos profissionais da saúde não apenas com a segurança do paciente, mas também com a sua própria segurança. No que diz respeito à segurança do paciente, destacam-se os itens de checagem de sinais vitais, procedimentos, ventilação e medicação. A avaliação da condição clínica do paciente a partir do checklist está em consonância com o fluxograma do Practical Approach to Care Kit (PACK) ${ }^{12}$, o qual norteia o atendimento desses pacientes atendidos na APS do município e auxilia na classificação da gravidade dos casos. No tocante à segurança dos profissionais, o checklist se constitui pelo item de checagem "Biossegurança", com cuidados relativos ao ambiente e uso de EPI, como a limpeza de equipamentos, a desinfecção terminal da sala e a troca da máscara, respectivamente, também descritos no PACK ${ }^{12}$.

$\mathrm{Na}$ terceira versão do checklist, além da inclusão de itens considerados importantes pela equipe para determinar a evolução da condição clínica, como o horário da checagem de sinais vitais e HGT, foram inseridas orientações de isolamento domiciliar dos contatos familiares da pessoa suspeita de COVID-19, com vistas à proteção comunitária e mitigação do vírus. As medidas para minimizar a circulação de pessoas são fundamentais na diminuição dos níveis de transmissão do novo coronavírus, distribuindo os atendimentos no tempo e evitando assim a propagação acelerada do vírus e o consequente colapso do sistema de saúde ${ }^{8}$. A literatura corrobora o papel da Enfermagem no controle da transmissão viral, na as- 
sistência às pessoas com COVID-19 e nas orientações à comunidade, destacando-se o olhar atento ao cuidado do ser humano, do ambiente, da família e coletividade, com empatia e acolhimento ${ }^{13}$

Com relação à implementação do checklist proposto, considerou-se o mesmo de fácil aplicação, por proporcionar segurança na garantia de que nenhum item seja negligenciado no cuidado, e melhora a comunicação entre os profissionais envolvidos no atendimento. Julga-se que estes benefícios na implementação do instrumento têm relação com a sua forma de apresentação final, dividido em itens a serem checados antes da transferência do paciente pelo SAMU: sinais vitais, procedimentos, biossegurança, ventilação, medicação, alergia medicamentosa, cuidados com a família, comunicação com outros pontos da rede de atenção às urgências e observações. Nesse sentido, preconiza-se que as ferramentas de comunicação devem ser claras, precisas e objetivas, além de incluir todas as informações necessárias para garantir a eficácia das ações e o cuidado contínuo'.

Ressalta-se que checklist são instrumentos empregados para auxiliar o desenvolvimento de rotinas complexas, de modo a aumentar a segurança, diminuir gastos e otimizar o tempo da equipe 6 . A Enfermagem tem fortalecido o cuidado organizando suas práticas a partir de checklis$\mathrm{ts}^{14}$. Considerando-se que uma pandemia desta magnitude nunca fora antes vivenciada pelos profissionais da saúde, entende-se que sistematizar a assistência de Enfermagem pela elaboração de uma ferramenta de comunicação como o checklist apresentado, tem enorme importância no contexto do enfrentamento desta situação.

Por fim, a Enfermagem brasileira e mundial vivencia um momento singular face à pandemia COVID-19, momento de se reinventar diante de um cenário desafiador, com atenção à saúde do trabalhador e à segurança do paciente ${ }^{13}$, a exemplo da proposta descrita no presente estudo. Para tanto, destaca-se ainda como fundamental a garantia de condições de trabalho para que os profissionais de Enfermagem ofereçam assistência de qualidade ${ }^{15}$ e encontrem estratégias de preservação da vida diante da pandemia, o que é requerido do mundo inteiro ${ }^{16}$.

\section{PRINCIPAIS RESULTADOS ALCANÇADOS}

Considera-se que a aplicação da ferramenta concedeu ao profissional enfermeiro e demais membros da equipe de APS maior segurança no cuidado, pois possibilitou acessar informações de forma rápida, com a garantia de que nenhum dado fosse negligenciado. Essa sistematização da coleta de dados por meio do checklist, favoreceu o diálogo entre os profissionais médico e enfermeiro durante o atendimento, assim como a comunicação com a família e com os outros pontos da RAU. Ainda, otimizou o atendimento e proporcionou maior eficiência na utilização de recursos fundamentais no atendimento de pessoas em situação de urgência. Desse modo, entende-se que a experiência a que se refere este estudo foi exitosa pelos resultados alcançados com o checklist proposto.

\section{LIMITAÇÕES DA EXPERIÊNCIA}

A experiência se limitou à elaboração e implementação do checklist por equipes de Saúde da Família de um CS, o qual foi aplicado no cuidado de uma pessoa suspeita de COVID-19 com sinais de gravidade.

\section{CONTRIBUIÇÕES PARA A PRÁTICA}

Considerando-se a aplicabilidade do checklist proposto, a experiência relatada poderá contribuir sobremaneira na assistência de enfermagem em situações semelhantes e, quiçá, proporcionará resultados favoráveis à pessoa suspeita do novo coronavirus com sinais de gravidade e equipe de saúde na APS, no que se refere à segurança e biossegurança, respectivamente.

\section{CONSIDERAÇÕES FINAIS}

A elaboração coletiva do checklist para o cuidado à pessoa suspeita de COVID-19 com sinais de gravidade na APS supriu a necessidade dos profissionais de realizarem a atenção à urgência a esse público com mais qualidade e segurança. Sua implementação demonstrou se tratar de um instrumento claro e objetivo, embasado em orientações técnicas e direcionado ao atendimento de pessoas com sintomatologia característica da infecção pelo novo coronavirus. Julga-se o checklist relevante, mas estudo de validação de conteúdo deve ser desenvolvido com brevidade, a fim de aprimorar o mesmo para aplicação na assistência.

Contribuição dos Autores: Patrícia Madalena Vieira Hermida - concepção e/ou desenho do estudo; coleta, análise e interpretação dos dados; redação e/ou revisão crítica do manuscrito; aprovação da versão final a ser publicada. Natálie D’Avila da Silveira - coleta, análise e interpretação dos dados; redação do manuscrito. Lucas José Bringhenti - coleta, análise e interpretação dos dados; redação do manuscrito. Tatiane Shimanko Bugs - coleta, análise e interpretação dos dados; redação do manuscrito. Paola Miotto - redação do manuscrito. Meriane Ferrarezi Chiari - redação do manuscrito. Paola Miranda Sulis - redação do manuscrito. 


\section{REFERÊNCIAS}

1. Shereen MA, Khan S, Kazmi A, Bashir N, Siddique R. COVID-19 infection: origin, transmission, and characteristics of human coronaviruses. J Adv Res [Internet]. 2020 [cited 2020 Jul 16]; 24(1):91-98. Available from: https://www.ncbi.nlm. nih.gov/pmc/articles/PMC7113610/pdf/main.pdf.

2. Ministério da Saúde (BR). Portaria GM/MS n. 188, de 3 de fevereiro de 2020. Declara Emergência em Saúde Pública de importância Nacional (ESPIN) em decorrência da infecção humana pelo novo coronavírus (2019-nCoV). Diário Oficial da União, Brasilia (DF), 2020 fev 4 [cited 2020Jun 16]. Seção 1:1. Available from: http://www.in.gov.br/en/web/ dou/-/portaria-n-188-de-3-de-fevereiro-de-2020-241408388.

3. Ministério da Saúde (BR). Protocolo de manejo clínico do coronavírus (COVID-19) na atenção primária à saúde. [Internet].2020. [cited2020Jun16].Availablefrom:https://www.saude.gov.br/images/pdf/2020/marco/20/20200318ProtocoloManejo-verO02.pdf

4. Sistema Conselho Federal de Enfermagem (COFEN)/Conselhos Regionais em Foco. Nota Técnica no 01/2020. Orientações sobre o novo coronavírus (COVID-19). Enferm Foco [Internet] 2020 [cited 2020 Jul 16]; 11(1):8-9. Available from: http://revista.cofen.gov.br/index.php/enfermagem/article/view/4089/694.

5. Brandão MAG, Barros ALBL, Primo CC, Bispo GS, Lopes ROP. Nursing theories in the conceptual expansion of good practices in nursing. Rev Bras Enferm [Internet]. 2019 [cited 2020 Jul 16]; 72(2):577-581. Available from: https://doi. org/10.1590/0034-7167-2018-0395.

6. Bueno BRM, Moraes SS, Suzuki K, Gonçalves FAF, Barreto RASS, Gebrim CFL. Characterization of handover from the surgical center to the intensive care unit. Cogitare Enferm [Internet]. 2015 [cited 2020 Jun 16]; 20(3):511-517. Available from: https://revistas.ufpr.br/cogitare/article/view/40274/26257.

7. Secretaria Municipal de Saúde de Florianópolis (SC). Diretoria de Atenção em Saúde. Gerência de Integração Assistencial. Orientações para manejo do paciente com suspeita de COVID-19 e com sintomas respiratórios graves na APS. Florianópolis: Secretaria Municipal de Saúde; 2020.

8. Portela MC, Pereira CCA, Lima SML, Andrade CLT, Soares FRG, Martins M. Nota Técnica 1: limites e possibilidades dos municipios brasileiros para o enfrentamento dos casos graves de Covid-19. [Internet]. 2020 [cited 2020 Jul 16]. Available from: https://portal.fiocruz.br/documento/nota-tecnica-limites-e-possibilidades-dos-municipiosbrasileiros-para-o-enfrentamento-dos.

9. Ministério da Saúde (BR). Secretaria de Atenção à Saúde. Departamento de Atenção Especializada. Regulação médica das urgências [Internet]. Brasilia: Editora do Ministério da Saúde; 2006 [cited 2020 Jun 16]. Available from: http://bvsms.saude.gov.br/bvs/publicacoes/regulacao_medica_urgencias.pdf.

10. Hermida PMV, Nascimento ERP, Belaver GM, Danczuk RFT, Alves DLF, Jung W. Perception of family health teams regarding primary care provided in the urgency network. Rev Enferm UFPE online [Internet]. 2016 [cited 2020 Jun 16]; 10(4):1170-1178. Available from: http://dx.doi.org/10.5205/reuol.8464-74011-1-SM.1004201601.

11. Nobrega DM, Bezerra ALD, Sousa MNA. Conhecimentos, atitudes e práticas em urgência e emergência na atenção primária à saúde. CED - Revista Eletrônica da Fainor [Internet]. 2015 [cited 2020 Jul 16]; 8(2):141-157. Available from: http://srv02.fainor.com.br/revista237/index.php/memorias/article/viewFile/386/254

12. Secretaria Municipal de Saúde de Florianópolis, Gerência de Integração Assistencial, Departamento de Gestão da Clínica. PACK COVID-19: Guia para profissional de saúde da Atenção Primária. 2. ed. Florianópolis, SC: Secretaria Municipal de Saúde de Florianópolis; 2020. Disponivel em: bit.ly/packfloripacovid19.

13. Miranda FMA, Santana LL, Pizzolato AC, Saquis LMM. Working conditions and the impact on the health of the nursing professionals in the context of covid-19. Cogitare enferm [Internet]. 2020 [cited 2020 Jul 16]; 25:e72702. Available from: https://revistas.ufpr.br/cogitare/article/view/72702/pdf_en.

14. Girondi JBR, Bússolo P, Rosa LM, Amante LN, Sebold LF, Gelbeck FL, et al. Validação de conteúdo de checklist de intervenções de enfermagem pré-operatórias para angioplastia. Enferm Foco [Internet] 2020 [cited 2020 Jul 31]; ll(2):11-17. Available from: http://revista.cofen.gov.br/index.php/enfermagem/article/view/2752/761

15. Santos VC, Persegona MFM, Souza EF, Almeida WC, Filete M, Silva MCN. Comitê Gestor de Crise do Coronavírus no âmbito do COFEN. Enferm Foco [Internet] 2020 [cited 2020 Jul 31]; 11(2):6-10. Available from: http://revista.cofen.gov. br/index.php/enfermagem/article/view/4213/763

16. Lourenção LG. A COVID-19 e os desafios para o sistema e os profissionais de saúde [Editorial]. Enferm Foco [Internet] 2020 [cited 2020 Jul 31]; 1l(1). Available from: http://revista.cofen.gov.br/index.php/enfermagem/article/ view/3488/726 